\title{
Investigation of the Jet Liquid Flows Structure by Multicolor Particle Image Velocimetry
}

\author{
S. S. Usmanova ${ }^{1}$, A. V. Kuchmenko², N. M. Skornyakova3, M. V. Sapronov4 \\ National research University Moscow power engineering Institute «MPEI» \\ ${ }^{1}$ ORCID: oooo-0oo1-7674-5103, shirinusmanova25@mail.ru \\ 2 ORCID: 0ooo-0003-1004-0252, ann.131@yandex.ru \\ 3 ORCID: oooo-0002-2919-6428,nmskorn@mail.ru \\ 4 ORCID: 0000-0002-8600-2036, maks-sapronov@yandex.ru
}

\begin{abstract}
The work aims investigating the application aspects of multicolor particle image velocimetry for the diagnosis of jet liquid flows. The main feature of the method proposed in this work compared to particle image velocimetry is the radiation probing with three laser planes of different wavelengths located at a short distance from each other. This modification allows obtaining information about the flow structure simultaneously in three different planes. A jet liquid stream from a sprayer has been considered as a research object. As a result, a laboratory setup was assembled and a series of experiments were carried out. The paper presents examples of experimental images, the processing result, describes its algorithm, and also estimates the accuracy of the data obtained.

Keywords: flow velocity, multicolor particle image velocimetry, particle image velocimetry.
\end{abstract}

\section{Introduction}

Recently, quite various types of injectors have found a broad application in a number of industries, for instance in chemical technologies, in fuel production, in heat and power engineering, etc. $[1,2]$. Injectors are also used in fluid rocket engines, vortex combustion chambers, cooling systems and burner devices [3 - 5].

The injectors based approach assumes that the information about stream structure and its main parameters such as speed and droplets sizes should first be taken into account. The lack of information about main nozzles parameters can lead to incorrect device operation and possibly to harmful consequences. For example, in the case of cooling systems, this can cause an unequal distribution of the coolant over the object surface and lead to decommission.

The operating principle of most existing systems for measuring the velocity of gas-fluid flows is based on optical methods. The main advantage of such techniques is noninvasiveness, remoteness and non-inertia. Notable among them are methods involving the use of laser radiation [6 - 8]. These techniques include Laser Doppler Velocimetry (LDV) and Particle Image Velocimetry (PIV).

Laser Doppler Velocimetry relies on the determination of frequency shift of radiation scattered by the studied medium. The method also has some other unique properties including high spatial resolution and ability to measure three components of the velocity vector. However, LDV allows obtaining the velocity values only at one point of space. In contrast to LDV, Particle Image Velocimetry allows the two components of flow velocity vectors to be measured simultaneously in a plane. For PIV measurements, investigated flow is illuminated by a laser sheet and the radiation scattered by particles is recorded. By the way, the most complete information about the flow can be obtained by PIV modifications such as stereo, tomography and multi-color PIV. 
This paper discusses the possibility of using multi-color Particle Image Velocimetry (MPIV) to diagnose flows from injectors.

\section{Multicolor particle image velocimetry}

Multicolor Particle Image Velocimetry significantly differs from the other existing PIV modifications. This method allows the vector velocity field in several planes simultaneously to be obtained. Based on this concept, it will be enough to use only one camera to obtain information about three-dimensional flow structure. From the assumption that when the experimental image is decomposed into color channels, the response from the corresponding laser plane will correspond to a certain color channel. To illuminate the flow volume, it is more expedient to use a laser radiation source than a natural one. However, it may also be difficult to find a laser that would generate light with the required wavelength range.

Results of measuring by multicolor PIV may not always be true, because at present there is no direct correspondence between the information contained in any color channel obtained by decomposing an experimental image into color components by digital methods and the information transmitted by an optical signal of the corresponding color. This is due to the use of a color digital camera as a detector, namely, its sensitivity property. The typical camera response curve is usually unequal. Figure 1 shows the method measurement scheme.

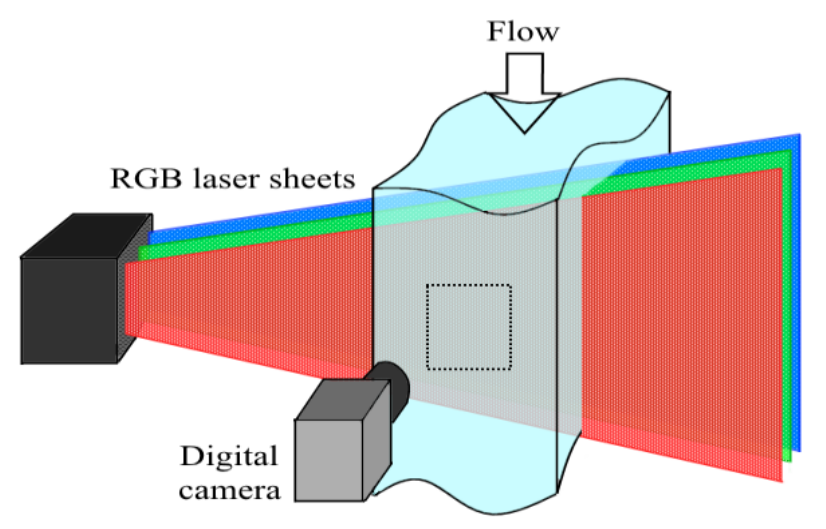

Fig. 1. MPIV measurement scheme

The principle operation illuminates the measured region by three RGB laser sheets and registers particles positions in scattered light at short time intervals.

Seeding particles must move at the flow speed and not introduce any disturbance. For this purpose, particles should be small, and their density should be close to the flow density. In the MPIV, red, green and blue laser planes located at a small distance from each other are used as probing radiation. Laser modules are selected in such way that when the experimental image is divided into three colors, the signal from each plane is present only in one of three color channels. Registration is carried out by a color digital camera. In turn, each image recorded will have three main colors. As a result, by applying the cross-correlation to processed images pairs for each color channel, it is possible to get information about velocities distributions in three planes. On the basis of this data, it is capable to visualize a flow three-dimensional velocity field.

\section{Influence of scheme parameters on reliability of results}

The accuracy of MPIV measurements can be affected by several factors. The three most important ones are the adjustment accuracy, the cross-correlation processing accuracy and the parameters of an experimental setup. The first factor doesn't strongly influence the accuracy of experimental data. Compared to other PIV modifications, the technique is quite simple to implement and doesn't require a complex precision adjustment. Thus, it is possible 
to minimize the adjustment error. The same is true for the second factor. The processing error in PIVview program is less than $3 \%$.

However, the measurements accuracy can be significantly affected by the installation parameters, such as the wavelength of the radiation source. Therefore, necessary requirements for the component base of the setup for MPIV measurements is the separation of the detected scattered radiation of red, green and blue colors according to the corresponding color receiving channels of the digital camera. Ideally, the scattered radiation from particles of one of the probing components should be registered for each of the three color components of an experimental image, for example, there should be no signal for the other two colors in the red color channel. To fulfil this requirement, we should take into account the spectral sensitivity of the photodetector when choosing radiation sources. Otherwise, there will be no correspondence between the physical process and experimental data.

The accuracy of MPIV measurements can also be affected by the ratio of radiation intensities of laser planes. The spectral sensitivity of the photodetector selected as the receiving equipment may be unequal. Then, as a result of the MPIV measurements, the response from the receiver for each plane to the radiation scattered by the same object will be different. A large difference in values of the receiver response for each of three laser planes can lead to incorrect experimental results. For example, let the radiation intensities of three RGB laser planes be equal, spectral sensitivity of the photo detector is uneven, in such way the response of the receiver to two laser planes will be much less than the response to the third plane. In this case, the receiver will not be able to register the entire signal from the first two laser planes The camera matrix is very likely to be illuminated when registering a signal from the third plane. As a result, the signal from this plane will be present not in one channel, but in all three.

Diagnosing vortex structures by MPIV method also requires choosing the correct distance between laser planes. For example, in case of diagnostics of the flow shown in Figure 2, it is advisable to arrange laser planes as follows (Figure 3).

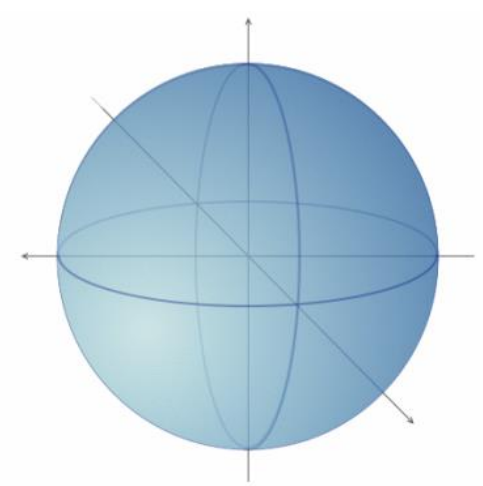

Fig. 2. Border of vortex structure

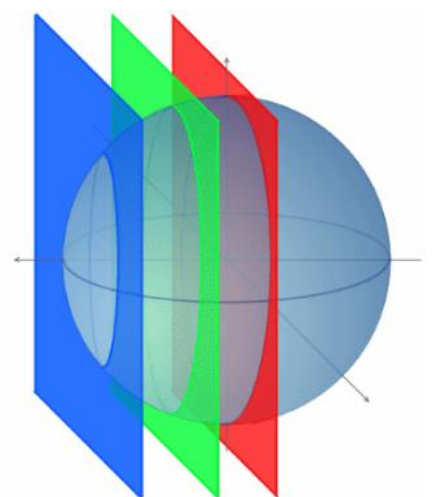

Fig. 3. Correct location of laser planes 
Otherwise, if laser planes are located at a small distance (Figure 4), the flow structure will not be fully visualized, because the selected area doesn't cover the entire flow structure.

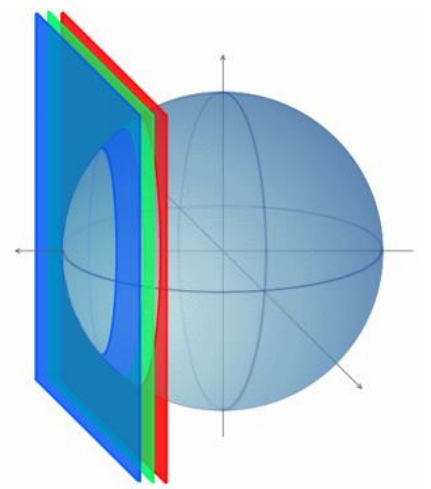

Fig. 4. Incorrect location of laser planes

Also, choosing the distance between laser planes requires taking into account a depth of camera field. If laser planes aren't located completely within the depth of the camera field, then part of the signal will be lost.

The other problem that occurs during the experimental setup is the incorrect configuration. Reconstructed coordinates of the particle's positions along $\mathrm{Z}$ axis may not correspond to their actual positions at a large (relative to diameter of particles) thickness. The obtained data indicate that the information about positions of tracer particles along $\mathrm{Z}$ axis within the thickness of a laser plane isn't taken into account when performing MPIV measurements. Therefore, when developing an experimental setup, it is advisable to strive for a minimum thickness of laser planes.

\section{Testing of the developed setup}

The developed setup has been tested on air bubbles inside the glass. During testing, the glass was previously fixed on a two-coordinate table with a micrometer screw. Positions of bubbles were recorded as the glass was moved in different directions.

In the first experiment, the glass was moved along the $\mathrm{x}$ axis with the same step equal to $0.5 \mathrm{~mm}$ from the position $x=0 \mathrm{~mm}$ to the position $x=9 \mathrm{~mm}$ (Figure 5).

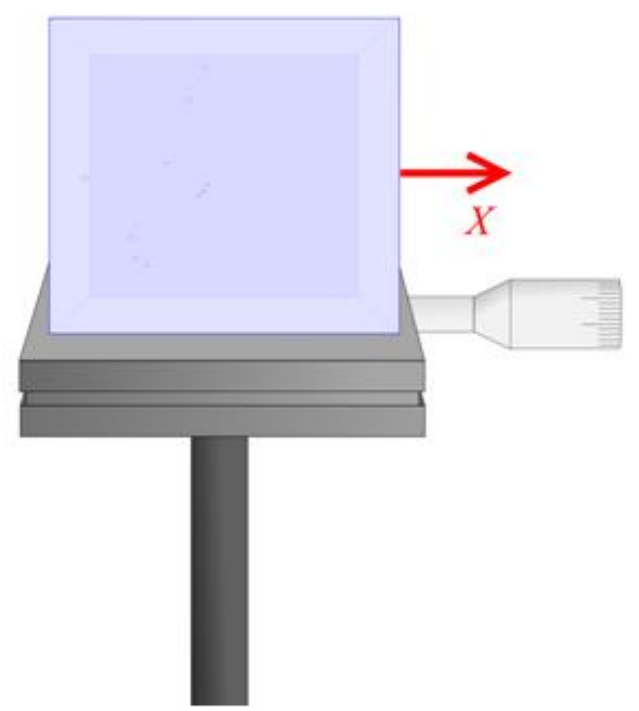

Fig. 5. Direction of glass displacement in first experiment 
During processing, experimental images were first decomposed into three color channels. Figure 6 shows an example of one of the received images. The numbers indicate the air bubbles that were used for further analysis.

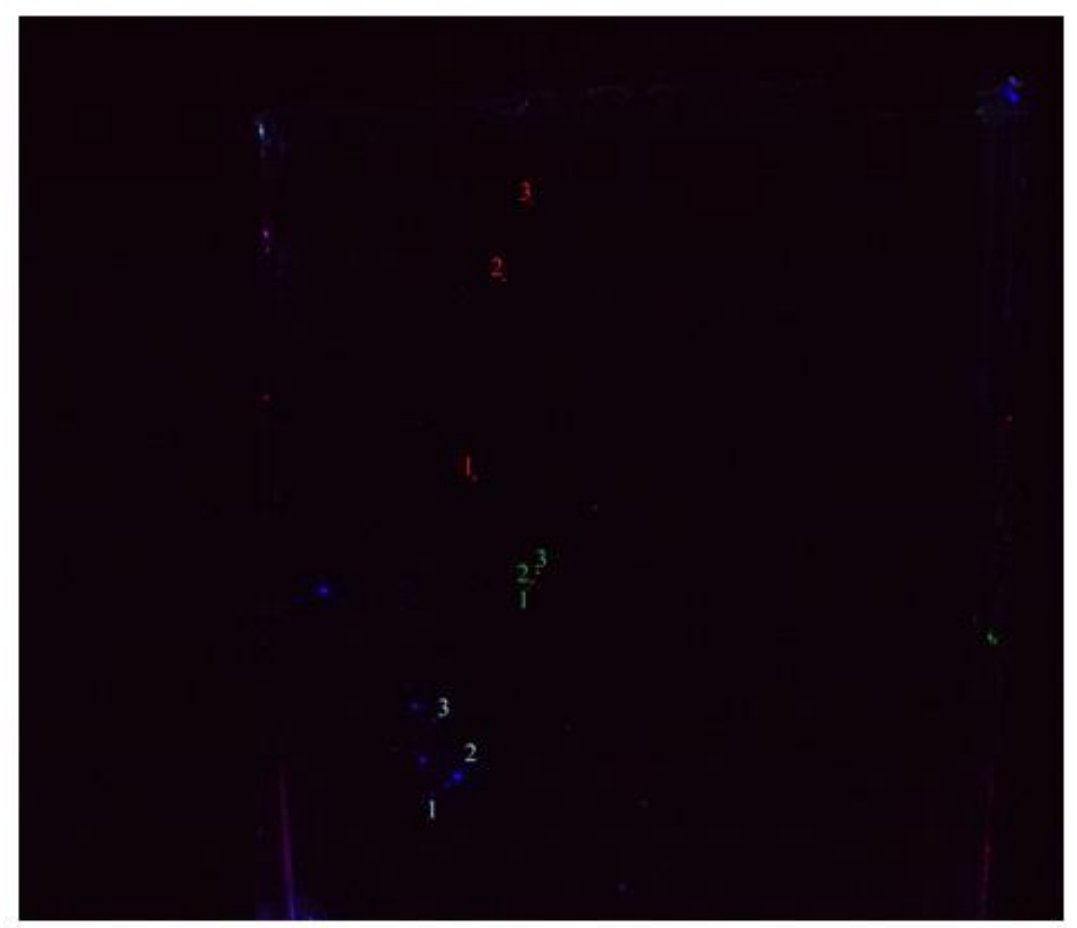

Fig. 6. Example of experimental image

Then, using the PIVview2CDemo program, we applied the cross-correlation processing to each pair of decomposed images and a vector field of displacements was constructed. According to processing results for each pair of experimental images, the value of displacement of selected air bubbles was determined for each color channel.

To assess the accuracy of data obtained, relative measurement error was calculated for each color channel using the following formula

$$
\delta=\frac{\left|x_{m}-x\right|}{x_{m}} \cdot 100 \%,
$$

where $x_{m}$ is the displacement value measured by a micrometer screw, $x_{t}=0.5 \mathrm{~mm} ; x$ is the displacement value measured by MPIV method.

The calculation results are presented in Table 1.

Table 1. Relative measurement errors for three channels

\begin{tabular}{|l|l|l|}
\hline Blue color channel & Red color channel & Green color channel \\
\hline $6,861 \% \approx 7 \%$ & $7,325 \% \approx 7 \%$ & $7,163 \% \approx 7 \%$ \\
\hline
\end{tabular}

During the second experiment, the glass was moved strictly along the $\mathrm{z}$ axis, and only one air bubble was recorded (Figure 7). 


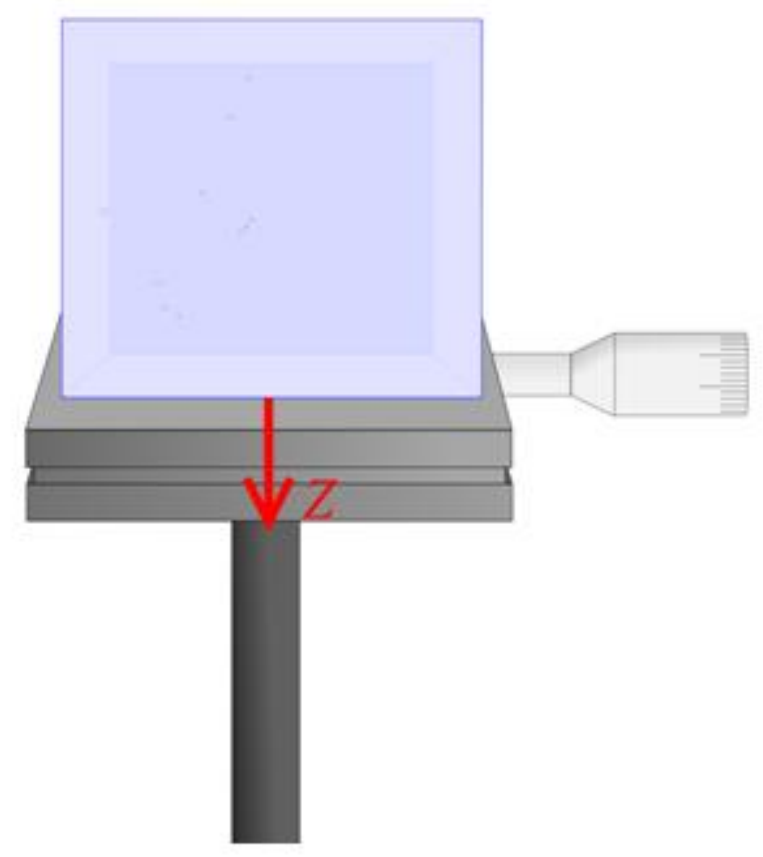

Fig. 7. Direction of glass displacement in second experiment

Figure 8 shows examples of experimental images.

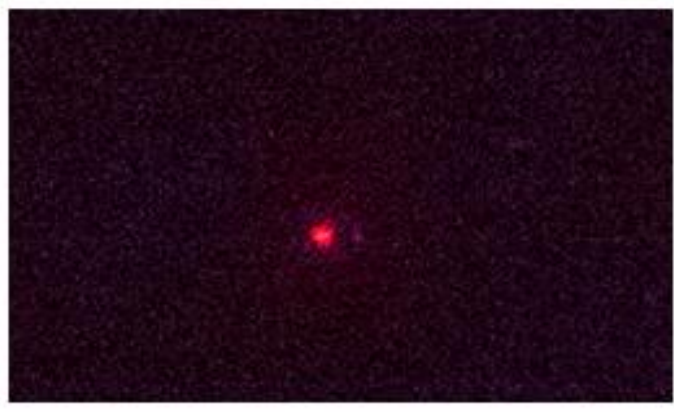

Red laser plane

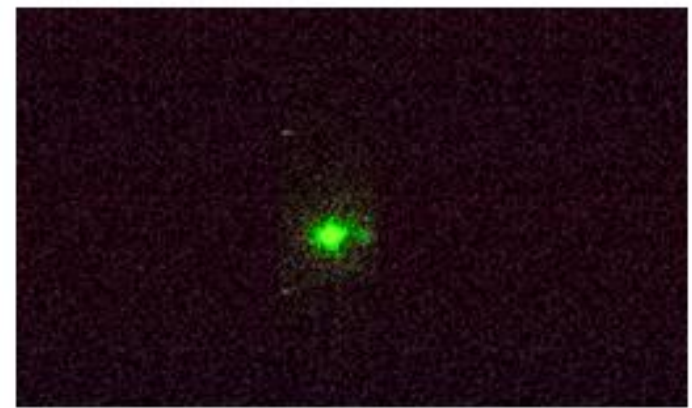

Green laser plane

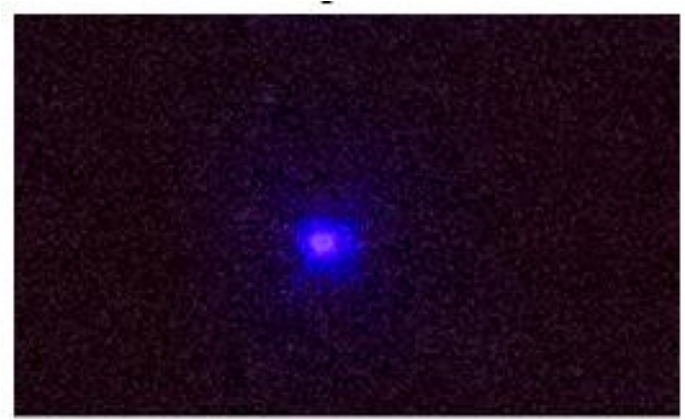

Blue laser plane

Fig. 8. Experimental images

To restore the object trajectory, a corresponding program was developed in MathCad. The algorithm is as follows. At the first stage, the experimental images are loaded into the program in the form of matrices with quantized pixel brightness values. Next, the center coordinates of the registered object are calculated for each of them. For this, we calculate the sum of the pixels brightness in the image with the following formula

$$
D=\sum_{i=0}^{m-1} \sum_{j=0}^{n-1} M_{i, j}
$$


where $m$ is the number of rows of the image matrix, $n$ is the number of columns, $M_{i, j}$ is the brightness value of the corresponding pixel.

After that, we compute the $x, y$ coordinates with the formulas below

$$
y=\frac{\sum_{i=0}^{m-1} \sum_{j=0}^{n-1}\left(j \cdot M_{i, j}\right)}{D} .
$$

$\mathrm{Z}$ coordinate is determined from experimental conditions. The trajectory of the object is constructed with the obtained coordinates $(x, y, z)$.

Figure 9 shows the reconstructed trajectory of the object for the second experiment.

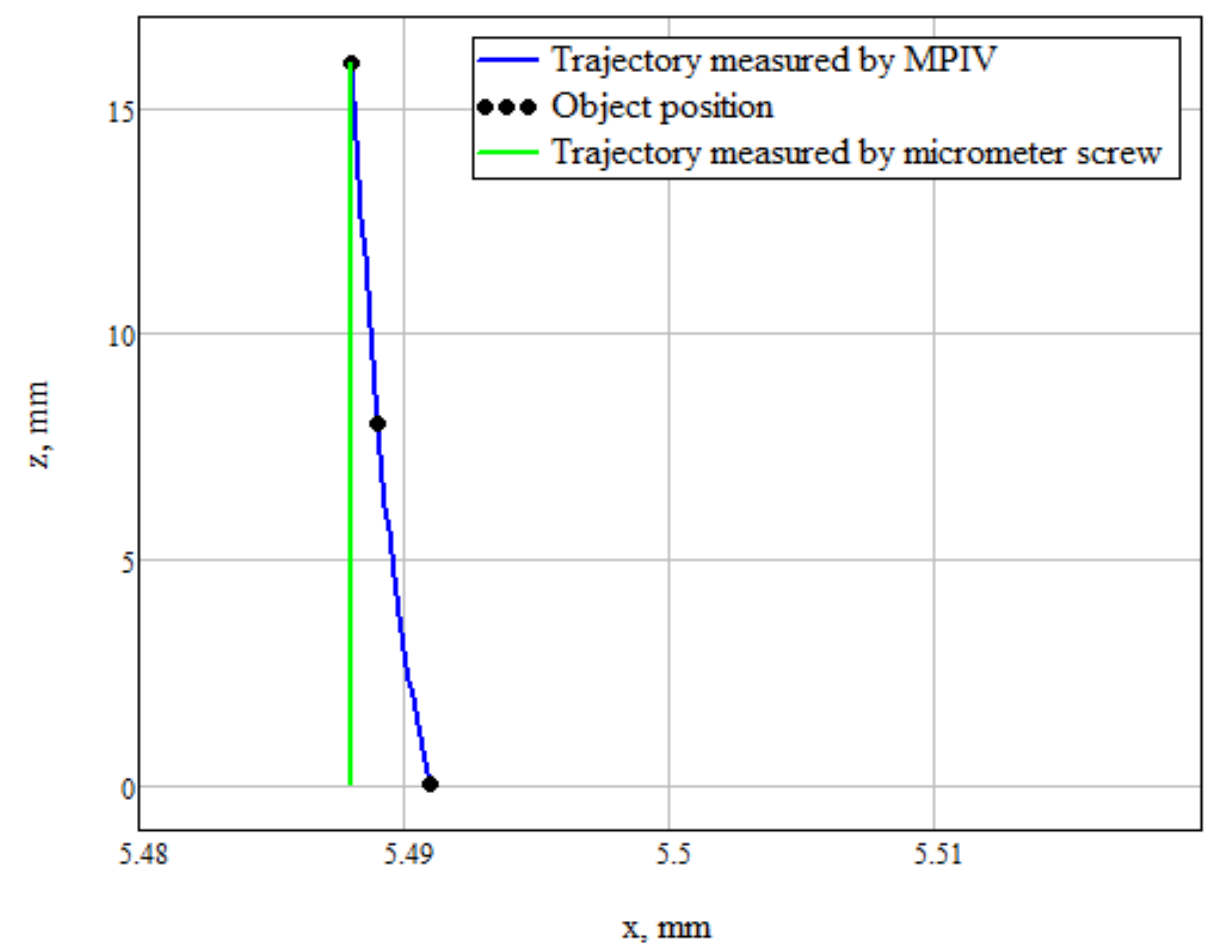

Fig. 9. Reconstructed trajectory of air bubble movement

To assess the results accuracy, the standard deviation (RMS) of the displacement value of the object center determined by MPIV method from the displacement value of the object center determined using a micrometer screw was calculated according to the following formula

$$
\sigma=\sqrt{\frac{\sum_{i=0}^{N-1}\left(S_{m}-S\right)^{2}}{N(N-1)}},
$$

where $S_{m}$ is the displacement value of the object center, measured by a micrometer screw, $\mathrm{S}$ is the displacement value of the object center, measured using MPIV

As a result, RMS was

$$
\sigma=0.033 \mathrm{~mm} .
$$

\section{Testing of the developed setup on a jet liquid flow}

During investigation, an experimental setup was developed. Its principle scheme is presented in Figure 10. 
The setup includes a set of three laser modules with wavelength $450 \mathrm{~nm}, 520 \mathrm{~nm}$ and 650 $\mathrm{nm}$; an optical system and a digital camera with color matrix.

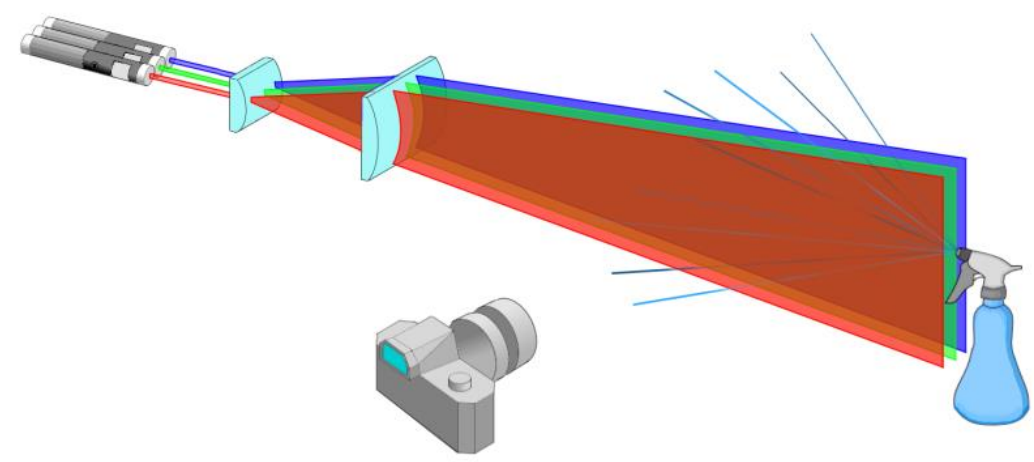

Fig. 10. Principle scheme of experimental setup

As a research object, the jet formed by the sprayer has been considered. Figures 11, 12 show one of the experimental images and its inverted version.

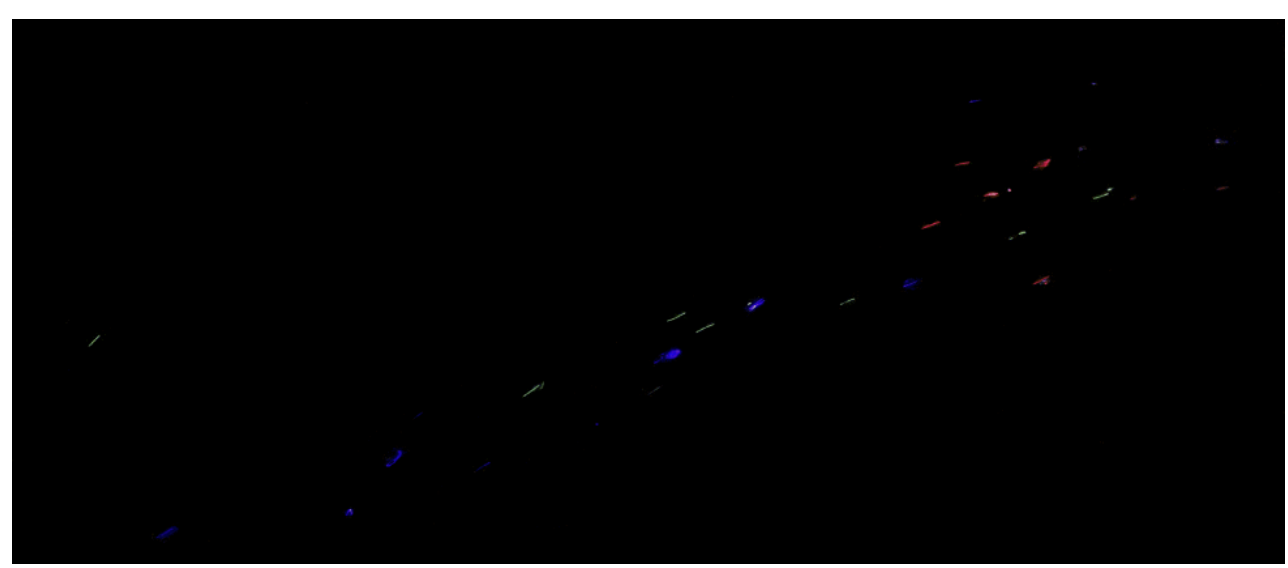

Fig. 11. Experimental image

Fig. 12. Inverted experimental image

By applying Particle Tracker Velocimetry (PTV) processing to images from each color channel, information about velocities of spray droplets in each laser plane can be obtained. Figure 13, 14 and 15 shows the result of processing. 


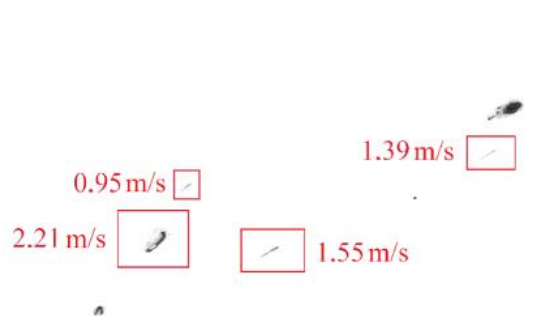

Fig. 13. Blue channel
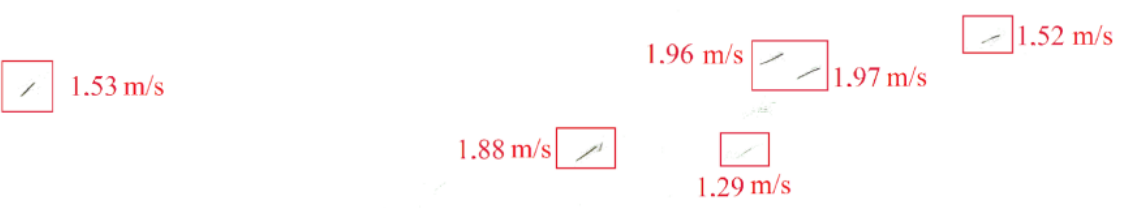

Fig. 14. Green channel

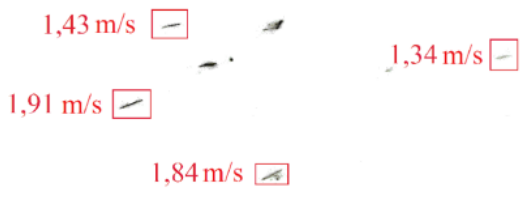

Fig. 15. Red channel

After that, the calculated speed values can be output from the program in form of a text file, and based on them, a three-dimensional velocity field can be constructed. 


\section{Conclusion}

The subject of this research work is the multicolor particle image velocimetry. Compared to the in contrast to other PIV modifications, the main advantage of the method proposed, is the ability to fix vector velocity fields simultaneously in several planes. This work investigates such important features of the process as the influence of wavelengths, the radiation intensity of laser planes, their thickness and the distance between them on the accuracy of MPIV measurements.

The developed setup allows measuring all three components of flow velocity vector in the range from $47 \cdot 4 \cdot 10-6 \mathrm{~m} / \mathrm{s}$ to $8.6 \cdot 10-3 \mathrm{~m} / \mathrm{s}$ and visualizing its three-dimensional structure. The measurement accuracy is $7-11 \%$. It should be noted that this speed range is due to the characteristics of the camera Nikon 1 J5. For the other camera used as the receiving optical system, for example, a high-speed camera, the speed range will be increased.

The setup can be widely used to solve problems in the field of aero-and hydrodynamics, visualization of fast-flowing processes and study of complex vortex structures. The final consumers of the proposed product are various rocket engineering enterprises, factories for cooling systems production, enterprises engaged in the development of fuel purification systems, aircraft engine manufacturers, research institutions.

\section{Acknowledgments}

The work was carried out within the framework of the project «Development of optical electronic setup for complex diagnostics of gas-liquid flows» with the support of the grant from the National Research University «MPEI» for the implementation of research programs «Electronics, radio engineering and IT» in 2020-2022.

\section{References}

1. Yu, W.; Yang, W.; Zhao, F. Investigation of internal nozzle flow, spray and combustion characteristics fueled with diesel, gasoline and wide distillation fuel (WDF) based on a piezoelectric injector and a direct injection compression ignition engine. Appl. Therm. Eng. 2017, 114, 905-920.

2. Yu, W.; Yang, W.; Mohan, B.; Tay, K.; Zhao, F.; Zhang, Y.; Chou, S.; Kraft, M.; Alexander, M.A.; Yong, A.; et al. Numerical and Experimental Study on Internal Nozzle Flow and Macroscopic Spray Characteristics of a Kind of Wide Distillation Fuel (WDF)-Kerosene 2016-01-0839. SAE Tech. Paper 2016.

3. Anacleto P. M., Fernandes E. C., Heitor M. V., Shtork S. I. 2015. Swirl flow structure and flame characteristics in a model lean premixed combustor // Combust. Sc. Te

4. Hreiz R., Gentric C., Midoux N., Laine R., Funfschilling D. Hydrodynamics and velocity measurements in gas-liquid swirling flows in cylindrical cyclones // Chemical Engineering Research and Design. 2014. Vol. 92. № 11. P. 2231-2246.

5. Yin J., Li J., Ma Y., Li H., Liu W., Wang D. Study on the Air Core Formation of a GasLiquid Separator // Journal of Fluids Engineering ASME. 2015. Vol. 137 / 091301. P. 1-9.

6. Kawaharada, N., Thimm, L., Dageförde, T., Gröger, K., Hansen, H., Dinkelacker, F. Approaches for detailed investigations on transient flow and spray characteristics during high pressure fuel injection // Applied Sciences (Switzerland)Volume 10, Issue 12, 1 June 2020, № 4410 .

7. Tekawade, A., Sforzo, B.A., Matusik, K.E., Fezzaa, K., Kastengren, A.L., Powell, C.F. Time-resolved 3D imaging of two-phase fluid flow inside a steel fuel injector using synchrotron X-ray tomography // Scientific ReportsVolume 10, Issue 1, 1 December 2020, № 8674.

8. Vacca, A., Hummel, S., Müller, K., Reichenbacher, M., Chiodi, M., Bargende, M. Virtual Development of Injector Spray Targeting by Coupling 3D-CFD Simulations with Optical Investigations // SAE Technical PapersVolume 2020-April, Issue April, 14 April 2020SAE 2020 World Congress Experience, WCX 2020; TCF CenterDetroit; United States. 